\title{
Lifi Based Audio Communication for Coal Mine Parameter Monitoring and Automatic Control System
}

\author{
P.Kalyana Sundaram, M.Anto Bennet, Giridhar Reddy
}

\begin{abstract}
Li-Fi (Light Fidelity) is a quick and shoddy optical rendition of correspondence. The principle segments of this correspondence framework are a high power white LED which goes about as a correspondence source and a silicon Photo diode which demonstrates great reaction to noticeable wavelength locale filling in as the getting component. An essential factor while planning Li-Fi is Line of Sight (LoS). The LED can be turned on and off to create advanced series of Os. Information is coded in the light which changes into new information by shifting the glinting rate of the LED. Since the speed of the light is exceptionally quick, the transmitted yield is gotten as voice signs to the specialists at the coal mineshaft. Coal mining and oring includes the disclosure of coal and its troublesome works of extraction, notwithstanding its evacuation and deal in the generation of concrete industry. Most wounds occurring in the underground mines incorporates the falling of rocks, slips and blasts. Harmful gas is produced amid the season of mining and oring forms. The mining laborers are influenced by lung illness by breathing in residue and lethal gas in Coal mining condition. This data is implied to the specialists at the coal mineshaft from the higher authorities through Li-Fi as a voice motions in this work.
\end{abstract}

Keywords: Li-Fi (Light Fidelity), Line of Sight (LoS), optical wireless communications $(\mathrm{OWC})$, Visible light communications $(\mathrm{VLC})$.

\section{INTRODUCTION}

The security issues of coal mineshafts have step by step transformed into a noteworthy worry for the general public and country. Since 1900, more than 100,000 laborers have been executed in coal mineshafts in the U.S. (Alford, 1980) and a lot more have been harmed and crippled . Underground coal diggers are presented to a wide Range of peril including gas blasts, moving rock, falls and hardware and portable gear mishaps. Coal as an imperative wellspring of vitality in modern generation, it assumes a urgent job in the national economy. So the excavator wellbeing is critical factor to be considered. Directly digger have cap for motivation behind ensuring the head. In this undertaking to build up a keen head protector for screen the focus dimension of unsafe gases and risky occasion of coal mineshafts and transmit the information to the base station utilizing Zig honey bee. To screen the fixation dimension of hurtful gases present in coal mineshaft like $\mathrm{SO} 2, \mathrm{NO} 2, \mathrm{CO}$ and so on the semiconductor gas sensor are utilized. We are additionally considering the wellbeing state of individual utilizing fall identifier accelerometer. The evacuation of digger protective

Revised Manuscript Received on July 22, 2019.

Dr. P.Kalyana Sundaram, ECE Department, SAVEETHA School of Engineering, Chennai, India.

Dr.M.Anto Bennet, ECE Department, VEL TECH, Chennai, India

Giridhar Reddy, ECE Department, VEL TECH, Chennai, India cap is additionally a critical factor under thought. The breaking point change is utilized to recognize the excavator's wear the protective cap or not. All information is transmitted from head protector to base station utilizing Zigbee .The information at beneficiary side is transmitted on the web utilizing GSM. Cautioning excavators in a mine can be a troublesome procedure remembering the ordinary working conditions that are experienced in a mine. Underground mines are extremely dull spots and thusly the excavators use security head protectors with connectable mining lights. The hardware use in underground mines can make a great deal of commotion and vibrations, which are intensified by the confined conditions in the underground passages. The issue related with the clamor is that notice an excavator with a speaker, alert, vibration unit and LED framework when a kindred digger is encountering an unsafe occasion would most likely be futile as the digger would not hear the caution. It was in this manner chosen to actualize a framework that will caution the excavator by blazing the mining light a couple of times. Utilizing this notice technique has the additional advantage of utilizing the mining protective cap light of the excavator who is encountering the perilous occasion. Glimmering the light continually at the same time show who is encountering the issue just as demonstrate the area of the digger. Likewise earphone and speaker are utilized to send the crisis message signal[1-4]. In our work we are recording diverse sound documents in APR. The voice or sound that must be recorded in APR is recorded with the inbuilt mouthpiece in that IC. Diverse changes are utilized to store distinctive sound documents. APR is constrained by PIC 16F877A microcontroller for sending the sound information record sequentially to the LIFI transmitter module. The sound record gets transmitter when the LED of the transmitter module flicker. The collector part contains a LIFI beneficiary module which gets the sound record. The collector module contains a photodiode to identify the transmitted audio[5,6]. This got information is then sent to the speaker. To screen the focus dimension of destructive gases, semiconductor gas sensors are utilized. Because of any reason diggers tumbles down and lose awareness likewise legitimate treatment isn't given them around then, so number of excavators is passed on. To defeat this issue the framework give crisis caution to the director if individual tumble somewhere around any reason. A few laborers don't know for security and they are not wear head protector. A Limit switch was then used to effectively decide if a digger has evacuated his cap or not.

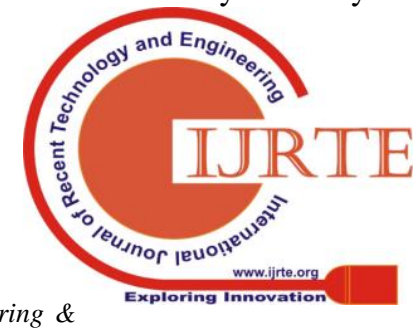

Blue Eyes Intelligence Engineering \& Sciences Publication 


\section{LIFI BASED AUDIO COMMUNICATION FOR COAL MINE PARAMETER MONITORING AND AUTOMATIC CONTROL SYSTEM}

This framework additionally gives an early cautioning, which will be useful to all excavators present inside the mine to spare their life before any loss happens. The framework utilizes Zigbee innovation and GSM for transmission of information. There is ready switch at recipient and transmitter side for crisis purpose[7-13].

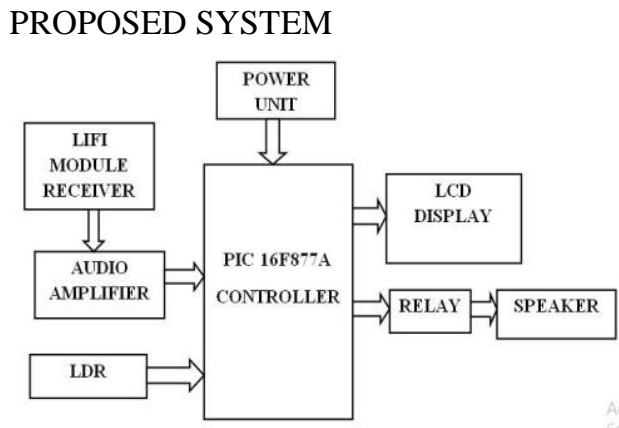

Fig: 1 Block Diagram Li-Fi data transmitter and receiver

Figure 1 demonstrates the square chart of Light Fidelity (Li-Fi) which is a bidirectional, fast and completely organized remote correspondence innovation like Wi-Fi. Obvious light correspondence and a subset of optical remote interchanges (OWC) and could be a supplement to RF correspondence (Wi-Fi or cell organizes), or even a substitution in settings of information broadcasting. It is wire and UV noticeable light correspondence or infrared and close bright rather than radio-recurrence range, some portion of optical remote interchanges innovation, which conveys significantly more data, and has been proposed as an answer for the RF-transfer speed restrictions. Obvious light interchanges (VLC) works by changing the current to the leds now and again at an extremely high rate, too fast to be in any way seen by the human eye. In spite of the fact that Li-Fi leds would need to be kept on to transmit information, they could be diminished to beneath human perceivability while as yet discharging enough light to convey information. The light waves can't enter dividers which makes an a lot shorter range, however increasingly secure from hacking, with respect to Wi-Fi. Direct observable pathway isn't vital for Li-Fi to transmit a flag; light reflected off the dividers can accomplish $70 \mathrm{Mbit} / \mathrm{s}$. Light Fidelity is a rapid remote conveying gadget which utilizes noticeable light as a medium Figure 2 demonstrates the transmitter and beneficiary segment of LIFI. LIFI set up includes a handset unit. LIFI information input (for example sequential information input) is given to the transmitter segment by methods for a PC from which the information is transmitted and gets got in a LIFI collector. The information which got gets intensified and get by methods for TTL yield.

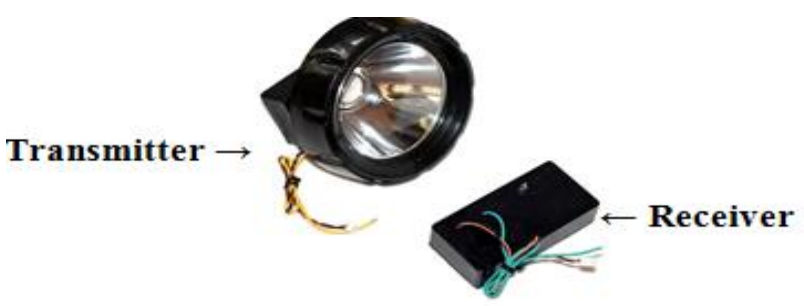

Fig.2 LIFI Transmitter and receiver

section

TEMPERATURE SENSORThe LM35 arrangement are exactness coordinated circuit temperature sensors, whose yield voltage is straightly relative to the Celsius (Centigrade) temperature. Figure .3 demonstrates the commonplace square graph of temperature sensor.

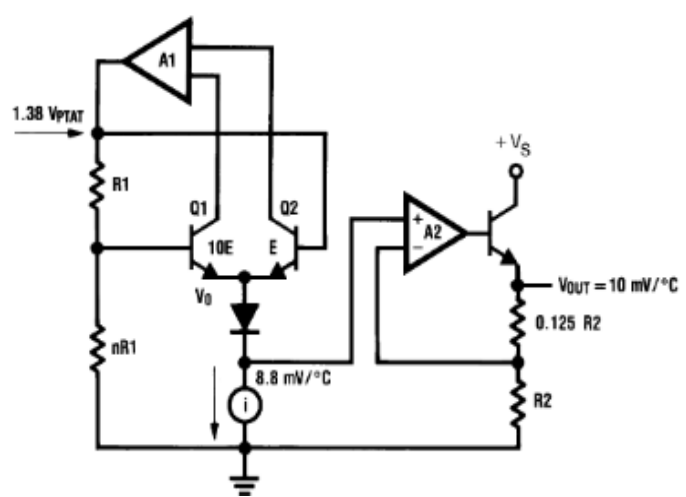

Fig3: Block Diagram of Temperature Sensor

\section{SINGLE VOICE RECORD/PLAYBACK MODULES}

The single voice board Figure appeared in 4 is a playback (ISD18B20), which is a solitary chip, single-message record/playback gadget. Accounts are put away into on-chip non-unstable memory, giving zero-control message stockpiling. With the installed Flash memory utilized, information maintenance as long as 100 years and ordinary 100,000 eradicate/record cycles can be come to. Time for chronicle is 8-20 seconds. Circle, run playback and single-pass playback highlights are available in this module. This module having amazing voice recording and high devotion replay. It very well may be utilized as a speaker module and can be controlled through microcontroller MCU.

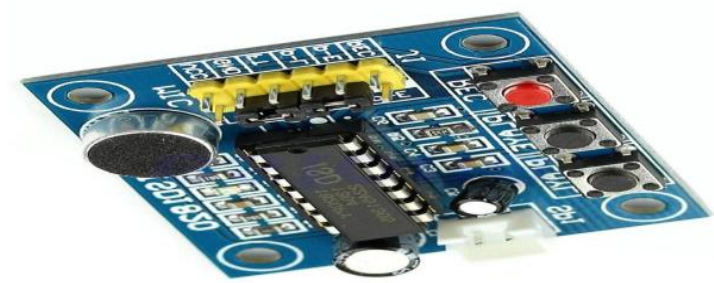

Fig 4: Single Voice Board

\section{GAS SENSOR MQ-2}

MQ-2 gas sensor appeared in Figure 5, which is structured with touchy material of sno2, which with lower conductivity in clean air. At the point when the objective ignitable gas exists, the sensor's conductivity is higher. Flag molding circuit is utilized to change over the difference in conductivity to compare yield motion with the info gas fixation. MQ-2 gas sensor has high affectability to LPG, Propane and Hydrogen, additionally could be utilized to Methane and other flammable steam, it is with minimal effort and reasonable for various application. 


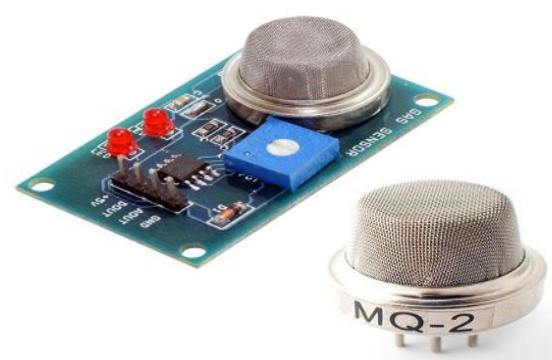

Fig5: Gas sensor MQ-2

\section{VIBRATION SENSOR}

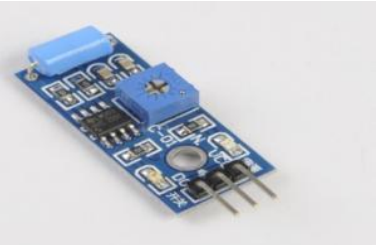

Fig 6: Vibration Sensor

The Vibration sensor module appeared in Figure 6 depends recognize whether there is any vibration that past the limit. the point when this no vibration, this module yield rationale LOW the flag show LED light, And the other way around. No stun, vibration switch was shut conduction state, yield of low yield, the green marker light. Stun, vibration switch immediately separated, the yield side yield high, the green light does not sparkle. The yield can be legitimately associated with the microcontroller through the microcontroller to recognize high and low, in this way distinguishing whether the vibration condition, assumed the job of the police.

\section{PIC Controller PIC 16F877A}

Different microcontrollers offer various types of recollections. EEPROM, EPROM, FLASH and so forth are a portion of the recollections of which FLASH is the most as of late created. Innovation that is utilized in pic16F877A is

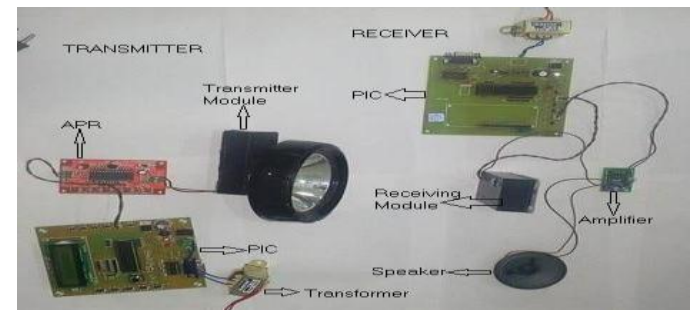

streak innovation, with the goal that information is held notwithstanding when the power is turned off. Simple Programming and Erasing are different highlights of PIC 16F877A. Figure 7 demonstrates the stick setup of PIC controller. on the vibration sensor SW-420 and Comparator LM393 to The limit can be balanced by the on-board potentiometer. At

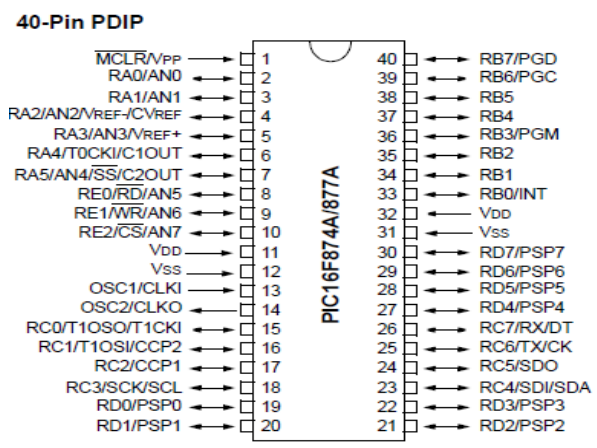

Fig 7: PIC 16F877A Pin diagram EXPERIMENTAL RESULTS

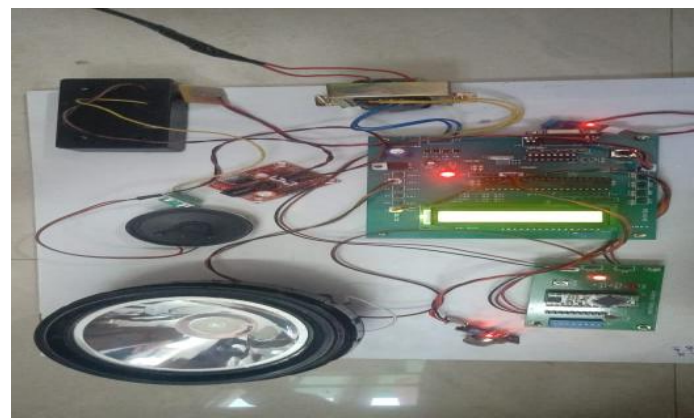

Fig $8::$ Image of the proposed system before transmitting

The information are first put away in the PIC controller and after that they are actualized. The given information are in the configuration of voice signals. At that point the voice signals are changed over into electrical flags and afterward changed over into double configuration. And after that they are permitted to transmit. Figure 8 demonstrates the picture of the proposed framework before transmitting.

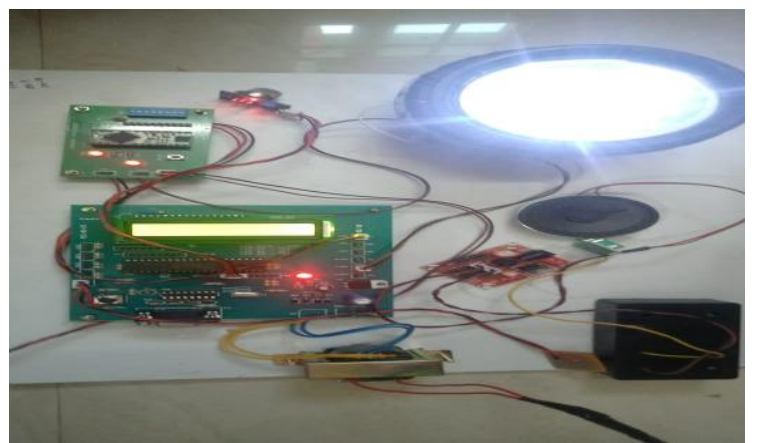

Fig 9:: Image of the proposed system during transmission

The twofold digits are then changed over into electrical signs. These electrical signs are then changed over into voice signals and the speaker gets the yield which is appeared in Figure 9. The parts that are available in the proposed framework are plainly given in the beneath referenced Figure 10.

Fig 10: Component Details

In this work we have utilized APR33A arrangement sound processor. It is a superior which records a decent quality voice. This processor is intended for straightforward key trigger, client can record and playback the recorded document. The one we have utilized comprises of 8 switches. Subsequently we can record 8 sound messages of around 20 sec each. The chip can be kept in shut down mode when

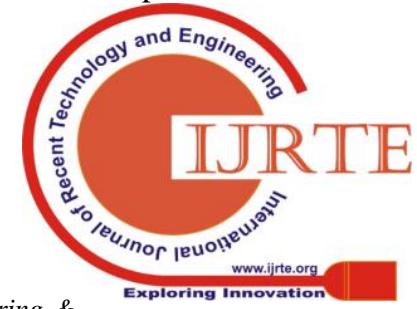




\section{LIFI BASED AUDIO COMMUNICATION FOR COAL MINE PARAMETER MONITORING AND AUTOMATIC CONTROL SYSTEM}

client does not have any desire to utilize it.

\section{CONCLUSION}

In any Mine, there are many hazard factors. So as to safe gatekeeper the general population working inside the mine its natural parameters are observed. In prior days if any unsafe impacts are happened send an individual and get report back. In our work we can maintain a strategic distance from this hazard by checking condition parameters. We can see the outcome at anyplace through IOT that what's going on in the mining. Li-Fi innovation is remote correspondence and eco-accommodating to nature. We can get the data with the assistance of LED (for example light). At that point send data to them to turn out from that point. In our work PIC controller is heart of our work, it controls all parameters. In our work, we can keep away from the medical issues that influence the mining laborers. On the off chance that an individual is influenced by any hazard factors, for example, high temperature, or any vibrations, hurtful gases. We can simply give them data in regards to the recognition of the previously mentioned variables. So in future with this module we can send a robot amid any catastrophe to make help the specialists to leave the mine.

\section{REFERENCES}

1. Revathi.S, Aishwarya. N J, Parana Sinha, Illakiya .K,(2018), "Audio Transmission Using LIFI”, ISSN:2454-132X, VOLUME.4, ISSUE.2

2. Rajesh H S, Punith D Gowda, Pallavi N.S, Shilpa. R, Kavitha B C,(2018), "Mining Environment System Using LIFI", ISSN:2278-0181

3. Sabditha Gauni, Manimegalai C.T, Kalimuthu K, Muralu Krishnan,(2018), "Voice Guidance System Using LIFI Technology", ISSN:1314-3395, Volume.118, No.20

4. S.R.Deokar, J.S. Wakode, (2017), "Coal Mine Safety Monitoring And Alerting Systems”, E-ISSN: n ,V. 2395-0056, Volume.4, Issue.3

5. K.H Shakthi Muruga Jacintha, Judysimon,(2017), "Safety System For Gold Mining Process Using Visible Light Communication" , ISSN:1311-8080, Volume.117, No.16

6. Humank, Kumawat, Shivam Verma, Prof.Subhabharathi .S, (2017), "Audio Transmission Through Visible Light Communication", ISSN: 2278-7798, Volume.6, Issue.5

7. Dr. AntoBennet, M, Sankar Babu G, Natarajan S, "Reverse Room Techniques for Irreversible Data Hiding", Journal of Chemical and Pharmaceutical Sciences 08(03): 469-475, September 2015.

8. Dr. AntoBennet, M , Sankaranarayanan S, Sankar Babu G, “ Performance \& Analysis of Effective Iris Recognition System Using Independent Component Analysis", Journal of Chemical and Pharmaceutical Sciences 08(03): 571-576, August 2015.

9. Dr. AntoBennet, M, Suresh R, Mohamed Sulaiman S, "Performance \&analysis of automated removal of head movement artifacts in EEG using brain computer interface", Journal of Chemical and Pharmaceutical Research 07(08): 291-299, August 2015.

10. Dr. AntoBennet, M “A Novel Effective Refined Histogram For Supervised Texure Classification", International Journal of Computer \& Modern Technology, Issue 01,Volume02,pp 67-73, June 2015.

11. Dr. AntoBennet, M, Srinath R,Raisha Banu A,"Development of Deblocking Architectures for block artifact reduction in videos", International Journal of Applied Engineering Research,Volume 10, Number 09 (2015) pp. 6985-6991, April 2015.

12. AntoBennet, $M \&$ JacobRaglend, "Performance Analysis Of Filtering Schedule Using Deblocking Filter For The Reduction Of Block Artifacts From MPEQ Compressed Document Images”, Journal of Computer Science, vol. 8, no. 9, pp. 1447-1454, 2012.

13. AntoBennet, M \& JacobRaglend, "Performance Analysis of Block Artifact Reduction Scheme Using Pseudo Random Noise Mask Filtering", European Journal of Scientific Research, vol. 66 no.1, pp.120-129, 2011. 